\title{
Locally Dispersing Populations in Heterogeneous Dynamic Landscapes with Spatiotemporal Correlations. II. Habitat Driven by Voter Dynamics
}

\author{
David E. Hiebeler ${ }^{\mathrm{a}, *}$, Jack L. Hill ${ }^{\mathrm{a}}$ \\ ${ }^{a}$ Department of Mathematics and Statistics, University of Maine, Orono, ME 04469
}

\begin{abstract}
We examine a spatially explicit population model on a dynamic landscape with suitable and unsuitable habitat driven by voter or contagion dynamics. We consider four cases, consisting of all combinations of local and global interactions for both population dispersal and habitat dynamics. For both local and global population dispersal, using local habitat dynamics always increases population density relative to the case with global habitat dynamics, due to the resulting segregation of habitat turnover, decrease in effective habitat turnover rate, and presence of stable habitat corridors. With global habitat dynamics, a population using local dispersal exhibits lower density than one with global dispersal due to local crowding as well as frequent disturbance due to habitat transitions. On the other hand, with local habitat dynamics, a population using local dispersal can exploit suitable habitat patches and use dynamic corridors to colonize new regions. The latter effect is not seen with static landscapes, where clustered habitat can lead to the isolation of suitable patches due to surrounding unsuitable habitat.
\end{abstract}

Keywords: habitat loss and fragmentation, cellular automata voter model, dynamic habitat, spatiotemporal structure, stochastic spatial simulation

\footnotetext{
* Corresponding author

Email address: hiebeler@math.umaine.edu (David E. Hiebeler)

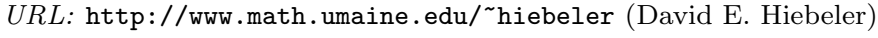

Preprint submitted to Elsevier

July 7, 2016

(C) 2016. This manuscript version is made available under the Elsevier user license http:/www.elsevier.com/open-access/userlicense/1.0/ 


\section{Introduction}

The relative importances of loss, fragmentation, and heterogeneity of habitat are widely known (Andrén, 1994; Bodin et al., 2006; Bonin et al., 2011; Debinski and Holt, 2000; del Castillo, 2015; Didham et al., 1998; Fahrig, 1997; Lande, 1987; Santos et al., 2015; Wiegand et al., 2005). Habitat heterogeneity in space and time has a wide variety of effects, including species composition in benthic communities (Munguia et al., 2011), seed production and dispersal and seedling establishment in forests (Uriarte et al., 2010), and the movements of organisms among patches (Collinge and Palmer, 2002; Diffendorfer et al., 1995). The effects of habitat heterogeneity may even vary over time within the lifetime of a particular organism (Bonin et al., 2011; Hovel and Lipcius, 2001).

Heterogeneities in space and time are intrinsically linked, with the variability in one dependent upon the scale within which the other is observed (Hiebeler and Michaud, 2012). Spatial and temporal variability, as well as their interactions, impact the structure of populations as well as communities (Chesson, 1985; Cleland et al., 2013; DeWoody et al., 2005; Fahrig, 1992; Jacquemyn et al., 2003; Johnson, 2004; Keymer et al., 2000; Loehle, 2007; Moloney and Levin, 1996; Snäll et al., 2005). For example, the spatial scale of disturbances influence species diversity, either by driving local extinction of species, or by leading to greater spatial variability in the densities of various species among communities (Limberger and Wickham, 2012).

Spatial or temporal variation are typically studied separately, although some investigations have addressed habitat that is both time-varying and spatially structured (Matlack, 2005; Matlack and Monde, 2004). It has been found that dynamic habitat can change the importance of features such as patch connectivity and patch quality, as compared with static habitat (Hodgson et al., 2009). 
In some cases, the effects of spatial features such as fragmentation are reversed in dynamic landscapes relative to static landscapes (Roy et al., 2004).

Temporal variation in habitat may arise from human land use, abiotic factors such as wind and rain, or the presence of other species that either promote or inhibit growth of a focal species. In all cases, the dynamics of habitat change may have a spatially local component. Here we explore the role of spatial scale of population dispersal and habitat dynamics on population density. Local habitat dynamics are modeled via a cellular automaton voter or contagion model. Variations of cellular automaton voter models have seen applications to the spread of Müllerian mimicry in predator-prey systems (Sherratt, 2006), maintenance of species diversity (Molofsky et al., 1999; Molofsky and Bever, 2002), as well as the emergence of large-scale patterns from small-scale interactions and decisions in social systems (Schelling, 2006). They are also a variation of the stepping stone models used in population biology and genetics (Kimura and Weiss, 1964; Weiss and Kimura, 1965; Renshaw, 1991). Here we use the voter model to simulate the spread of two variations such as neutral alleles, or equal competitors, that make up an important part of the substrate for the species of interest. It could also represent the spread of ideas about human land use decisions, for example the application of pesticides, herbicides, or fertilizers in residential plots.

\section{The Model}

We use a single-species patch occupancy model based on a continuous-time Poisson process on a discrete lattice where every site has $z$ neighbors. Our study used a rectangular lattice with $z=4$. Each site in the lattice is in one of three states: empty with suitable habitat (state 0), empty with unsuitable habitat (state 1), or occupied with suitable habitat (state 2). The habitat is dynamic, with transitions between suitable and unsuitable habitat following a 
cellular automaton voter or contagion model. Habitat dynamics and population dynamics were both explored using local and global neighborhoods.

Without loss of generality, we rescale the time units in the model so that each occupied site in the lattice updates its habitat state at rate 1 in the sense of a Poisson process, with times between updates following an exponential distribution with a mean of 1 . When a site updates its habitat, it copies the habitat state of one of its neighbors, chosen at random with equal probability for all neighbors. With the local neighborhood, the site to be copied is chosen from among the four adjacent sites in the lattice. With the global neighborhood, the site to be copied is chosen from among all sites in the lattice. If the habitat of an occupied suitable site changes to unsuitable, the site also becomes empty, i.e., the population is removed.

Each occupied site in the lattice reproduces at rate $\phi$ (with times between reproduction events following an exponential distribution with mean $1 / \phi$ ). When reproduction occurs, a target site is chosen from among one of the neighbors. Again, the four nearest neighbors are used with local dispersal, and all sites in the lattice are used with global dispersal. If the chosen target site is empty and suitable, it immediately becomes colonized; otherwise, the offspring is wasted. Finally, each occupied site becomes empty at mortality rate $\mu$.

Stochastic simulations were performed on a $300 \times 300$ lattice with wraparound boundary conditions. Throughout our study, we assume that the habitat distribution has reached a dynamic equilibrium, with the proportion and spatial correlations of suitable and unsuitable sites not changing other than due to stochastic fluctuations. When performing simulations, we first run each simulation until the habitat distribution has reached equilibrium We then introduce the population, setting proportion $\rho_{0}$ of suitable sites (chosen at random) as occupied. We used $\rho_{0}=\max (1-\mu / \phi, 0.1)$; this is the equilibrium density for a globally dispersing population with only suitable habitat (which serves as an 
overestimate of expected population density for our model), with a lower bound of $10 \%$ of sites initially occupied. Simulations were run until equilibrium was reached, using the same stopping criteria as Hiebeler et al. (submitted).

We explored four neighborhood combinations for landscape and population dynamics:

Case 1GhGp: global habitat dynamics, global population dispersal Case 2LhGp: local habitat dynamics, global population dispersal

Case 3GhLp: global habitat dynamics, local population dispersal

Case 4LhLp: local habitat dynamics, local population dispersal

Figure 1 displays images of $100 \times 100$ lattices corresponding to the four cases.

\section{Mathematical approximations}

Let $P[i]$ represent the proportion of sites in state $i$, with $i \in\{0,1,2\}$. Define $p_{s}=P[0]+P[2]$ as the proportion of sites containing suitable habitat; our assumption that the habitat distribution is at equilibrium implies that $p_{s}$ is fixed over time. Note that $P[0]+P[1]+P[2]=1$, giving $P[1]=1-p_{s}$ and $P[0]=p_{s}-P[2]$. We will summarize the state of the population via $\rho:=P[2] / p_{s}$, the proportion of suitable sites that are occupied.

Similarly, let $P[i j]$ represent the proportion of pairs of adjacent sites where the first site is in state $i$ and the second site is in state $j$. With three states per site, there are nine such pair probabilities. We assume rotational symmetry, eliminating three probabilities:

$$
P[10]=P[01], \quad P[20]=P[02], \quad P[21]=P[12] .
$$

The assumption that the habitat distribution is at equilibrium implies that the amount of unsuitable habitat $P[1]$ is fixed and that the spatial distribution 

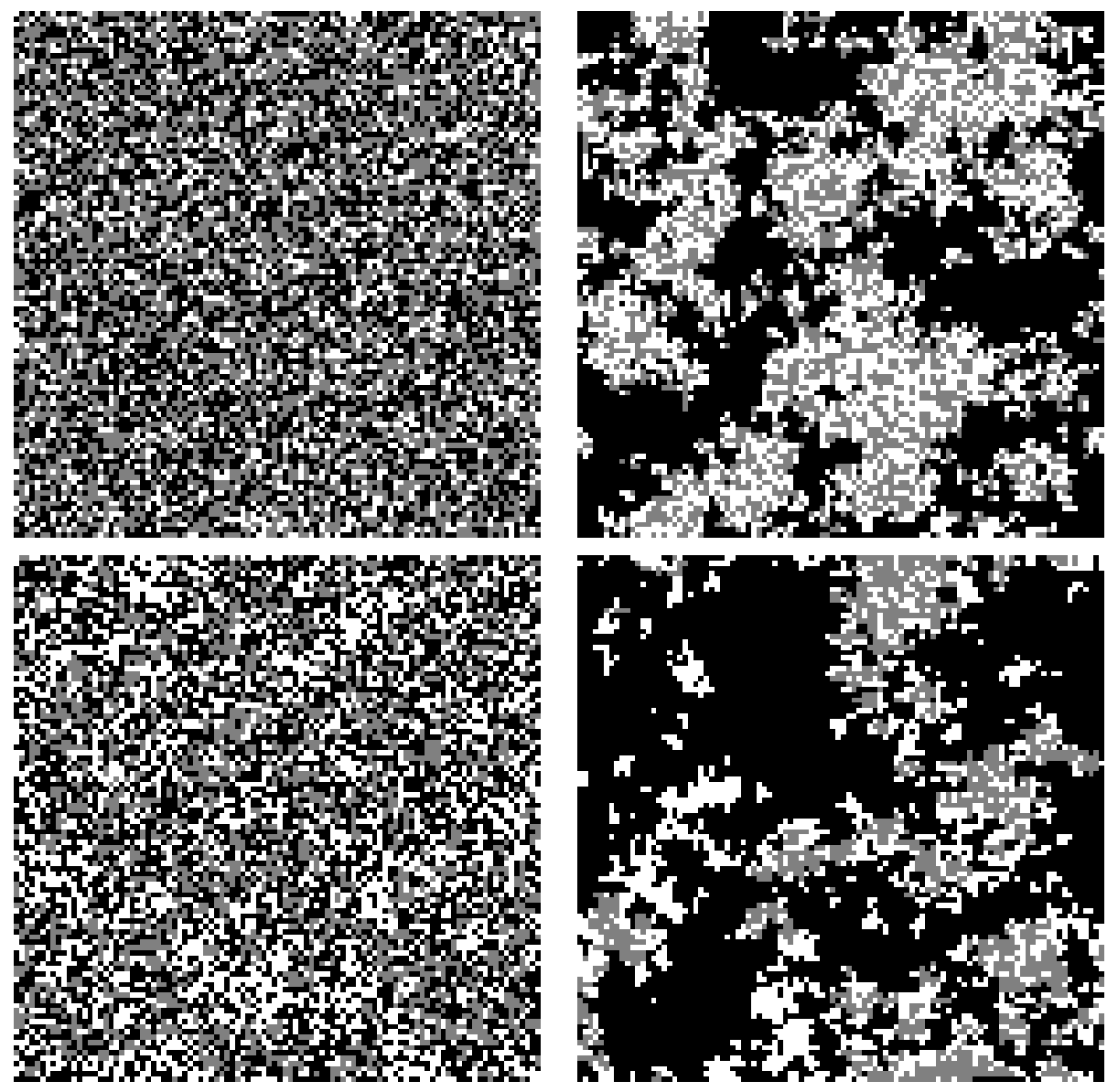

Figure 1: Images of landscapes with the four different neighborhood combinations. Empty suitable sites are colored white, unsuitable sites are black, and occupied suitable sites are gray. Top left: global population dispersal and habitat dynamics (case 1GhGp). Top right: global population dispersal, local habitat dynamics (case 2LhGp). Bottom left: local population dispersal, global habitat dynamics (case $3 \mathrm{GhLp}$ ). Bottom right: local population dispersal and habitat dynamics (case 4LhLp). With global habitat dynamics (cases 1GhGp and 3GhLp), fecundity $\phi=8$ was used. With local habitat dynamics (cases $2 \mathrm{LhGp}$ and $4 \mathrm{LhLp}$ ), $\phi=4$ was used. Mortality was $\mu=1$ for all four cases. 


\begin{tabular}{|c|c|c|}
\hline & Symbol & Meaning \\
\hline \multirow[t]{3}{*}{ States } & 0 & Empty suitable habitat \\
\hline & 1 & Empty unsuitable habitat \\
\hline & 2 & Occupied suitable habitat \\
\hline Model parameters & $\begin{array}{l}\phi \\
\mu \\
z\end{array}$ & $\begin{array}{l}\text { Per-capita reproduction rate } \\
\text { Per-capita mortality rate } \\
\text { Number of neighbors of each site in the lattice }\end{array}$ \\
\hline Probabilities & $\begin{array}{c}P[i] \\
P[i j] \\
Q_{i \mid j}\end{array}$ & $\begin{array}{l}\text { Probability a randomly-chosen site is in state } i \\
\text { Probability a randomly-chosen pair of adjacent sites are } \\
\text { in states } i, j \\
P[i j] / P[j] \text {, the probability that a randomly-chosen } \\
\text { neighbor of a randomly chosen state- } j \text { site is in state } \\
i \\
P[0]+P[2] \text {, the proportion of sites with suitable habitat } \\
P[2] / p_{s} \text {, the proportion of suitable sites occupied }\end{array}$ \\
\hline Model cases & $\begin{array}{l}1 \mathrm{GhGp} \\
2 \mathrm{LhGp} \\
3 \mathrm{GhLp} \\
4 \mathrm{LhLp}\end{array}$ & $\begin{array}{l}\text { Global habitat dynamics, global population dispersal } \\
\text { Local habitat dynamics, global population dispersal } \\
\text { Global habitat dynamics, local population dispersal } \\
\text { Local habitat dynamics, local population dispersal }\end{array}$ \\
\hline
\end{tabular}

Table 1: Symbols used in the differential equation model, and the four cases of the model studied here. 
of unsuitable habitat as characterized by $P[11]$ is also fixed. The fact that $\sum_{j \in\{0,1,2\}} P[j 1]=P[1]$ (with $P[1]=1-p_{s}$ fixed) eliminates another probability:

$$
\begin{aligned}
P[12] & =P[1]-P[01]-P[11] \\
& =1-p_{s}-P[01]-P[11]
\end{aligned}
$$

06 Finally, the constraint that all nine probabilities must sum to one eliminates one more:

$$
\begin{aligned}
P[22] & =1-P[00]-P[11]-2 P[01]-2 P[02]-2 P[12] \\
& =2 p_{s}-1+P[11]-P[00]-2 P[02] .
\end{aligned}
$$

This allows us to work with only the three pair probabilities $P[00], P[01]$, and $P[02]$, and recover the others as needed. However, we instead opt to use $P[2], P[00]$, and $P[01]$, and then use the fact that $\sum_{j \in\{0,1,2\}} P[j 0]=P[0]=$ $p_{s}-P[2]$ to recover

$$
P[02]=p_{s}-P[2]-P[00]-P[01] .
$$

We will also need conditional probabilities describing neighborhood configurations. We let $Q_{i \mid j}=P[i j] / P[j]$ be the probability that if we randomly choose a site in state $j$, then a randomly-chosen neighbor of that site is in state $i$. Similarly, $Q_{i \mid j k}$ represents the probability that for a randomly-chosen pair of adjacent sites in states $j$ and $k$, a randomly-chosen neighbor of the state- $j$ site (distinct from its known neighbor in state $k$ ) is in state $i$. We use ordinary pair approximation to replace

$$
Q_{i \mid j k} \approx Q_{i \mid j}=P[i j] / P[j]
$$
by assuming that the states of non-adjacent sites are independent when conditioned on the known state of a common neighbor they share. 
For the distribution of suitable and unsuitable habitat, we assumed proportion $p_{s}=0.5$ of sites were suitable in our study. Exploration of the model with other values of $p_{s}$ gave similar qualitative results. With global habitat dynamics, $Q_{1 \mid 1}=P[1]=0.5$ as well, due to the lack of spatial correlations. With local habitat dynamics, analysis of the voter model on a rectangular lattice gives a clustering coefficient of $Q_{1 \mid 1} \approx(2 P[1]+1) / 3=2 / 3$ when estimated using pair approximation (Hiebeler, 2007). We then use this to compute

$$
P[11]=P[1] Q_{1 \mid 1} \text {. }
$$

Measurements from simulations of the habitat distribution with local dynamics and with $p_{s}=0.5$ indicate $Q_{1 \mid 1} \approx 0.8079$, which will also be used with our differential equation models, and compared with the above approximated value of $2 / 3$. Note that the above values of $Q_{1 \mid 1}$ are referring only to clustering of habitat types, and do not include any information about the population.

For case 1GhGp (global habitat dynamics and population dispersal), we can write a differential equation for the proportion of sites that are suitable and occupied:

$$
\begin{aligned}
\frac{d P[2]}{d t} & =\phi P[2] P[0]-\left(\mu+r_{s \rightarrow u}\right) P[2] \\
& =\phi P[2]\left(p_{s}-P[2]\right)-(\mu+P[1]) P[2]
\end{aligned}
$$

where $r_{s \rightarrow u}$ is the rate at which suitable sites become unsuitable. With habitat dynamics using a global neighborhood, this rate is simply $P[1]$, the proportion of habitat that is unsuitable. The above ODE has the nontrivial equilibrium $P^{*}[2]=p_{s}-(\mu+P[1]) / \phi$, which gives

$$
\rho^{*}=1-\frac{\mu+P[1]}{p_{s} \phi}
$$

which is biologically meaningful as long as $\phi p_{s} \geq \mu+P[1]$, i.e., the effective reproduction rate (filtering out offspring that land on unsuitable sites) is greater 
than the total death rate due to both intrinsic mortality and the transition of suitable habitat to unsuitable habitat.

For case 2LhGp (local habitat dynamics, global population dispersal), the ODE for the proportion of suitable occupied sites is

$$
\begin{aligned}
\frac{d P[2]}{d t} & =\phi P[2] P[0]-P[2]\left(\mu+Q_{1 \mid 2}\right) \\
& =\phi P[2]\left(p_{s}-P[2]\right)-P[2] \mu-P[12] .
\end{aligned}
$$

ODEs for $P[00]$ and $P[01]$ (derived in Appendix A for this case as well as cases 3GhLp and 4LhLp) are

$$
\begin{aligned}
\frac{d P[00]}{d t}= & 2 P[01]\left(\frac{z-1}{z}\left(Q_{0 \mid 10}+Q_{2 \mid 10}\right)+\frac{1}{z}\right)+2 P[02] \mu \\
& -2 P[00]\left(\frac{z-1}{z} Q_{1 \mid 00}+P[2] \phi\right) \\
\frac{d P[01]}{d t}= & \frac{z-1}{z}\left(P[00] Q_{1 \mid 00}+P[02] Q_{1 \mid 20}+P[11]\left(Q_{0 \mid 11}+Q_{2 \mid 11}\right)\right)+P[12] \mu \\
& -P[01]\left(\frac{z-1}{z}\left(Q_{0 \mid 10}+Q_{2 \mid 10}+Q_{1 \mid 01}\right)+P[2] \phi+\frac{1}{2}\right) .
\end{aligned}
$$

${ }_{48}$ For case $3 \mathrm{GhLp}$ (global habitat dynamics, local population dispersal), the three ODEs are

$$
\begin{aligned}
\frac{d P[2]}{d t}= & P[0] Q_{2 \mid 0} \phi-P[2](\mu+P[1]) \\
\frac{d P[00]}{d t}= & 2 P[01](P[0]+P[2])+2 P[02] \mu-2 P[00]\left(P[1]+\frac{z-1}{z} Q_{2 \mid 00} \phi\right) \\
\frac{d P[01]}{d t}= & (P[00]+P[02]) P[1]+P[11](P[0]+P[2])+P[12] \mu \\
& -P[01]\left(P[0]+P[1]+P[2]+\frac{z-1}{z} Q_{2 \mid 01} \phi\right) .
\end{aligned}
$$

Finally, for case 4LhLp (local habitat dynamics and population dispersal), 
the ODEs are

$$
\begin{aligned}
\frac{d P[2]}{d t}= & P[0] Q_{2 \mid 0} \phi-P[2]\left(\mu+Q_{1 \mid 2}\right) \\
\frac{d P[00]}{d t}= & 2 P[01]\left(\frac{z-1}{z}\left(Q_{0 \mid 10}+Q_{2 \mid 10}\right)+\frac{1}{z}\right)+2 P[02] \mu \\
& -2 P[00] \frac{z-1}{z}\left(Q_{1 \mid 00}+Q_{2 \mid 00} \phi\right) \\
\frac{d P[01]}{d t}= & \frac{z-1}{z}\left(P[00] Q_{1 \mid 00}+P[02] Q_{1 \mid 20}+P[11]\left(Q_{0 \mid 11}+Q_{2 \mid 11}\right)\right)+P[12] \mu \\
& -P[01]\left(\frac{2}{z}+\frac{z-1}{z}\left(Q_{0 \mid 10}+Q_{2 \mid 10}+Q_{1 \mid 01}+Q_{2 \mid 01} \phi\right)\right) .
\end{aligned}
$$

Equations (1), (2), (3), and (4) are then used to close each of the systems of ODEs, which are analyzed numerically.

\section{Results}

We first performed a parameter sweep varying $\phi$ between 1 and 20, with fixed $\mu=1$. Figure 2 displays $\rho^{*}=P^{*}[2] / p_{s}$, the proportion of suitable sites occupied at equilibrium, as a function of $\phi$. Consider case 1GhGp (global habitat dynamics and population dispersal) as the baseline case. Case 2LhGp, with local habitat dynamics, has a higher equilibrium population density, despite the fact that the population is dispersing globally. This is due to the fact that with local habitat dynamics, suitable sites tend to have more suitable sites nearby, and therefore become unsuitable at a lower rate. Specifically, with $P[1]=0.5$ (half of the sites unsuitable), with global habitat dynamics, a suitable site becomes unsuitable at rate 0.5 . With local habitat dynamics, a suitable site becomes unsuitable (and vice versa) at rate $Q_{1 \mid 0} \approx 1-0.8079=0.1921$ (as measured from simulations). Relative to case $2 \mathrm{LhGp}$, case $4 \mathrm{LhLp}$ (with population dynamics now local as well) has an even slightly higher population density. Local population dynamics leads to more intraspecific competition for space due to local crowding, which typically decreases population density (Hiebeler, 2004). However, the higher chance of finding suitable habitat when reproducing locally 
rather than globally is enough to overcome the population crowding. Finally, case 3GhLp (global habitat dynamics, local population dynamics) has both the lowest population density and largest variability, as the population suffers from both local crowding and rapid habitat turnover.

Figure 3 also displays the results of the parameter sweeps, but this time with predicted population density from the various ODE approximations. For local habitat dynamics, results using $Q_{1 \mid 1}=2 / 3$ (from pair approximations) as well as $Q_{1 \mid 1}=0.8079$ (from simulation measurements) were used; for global habitat dynamics, $Q_{1 \mid 1}=P[1]=0.5$ was used. For case 1 GhGp, with no spatial correlations, simulations and predictions were in agreement. No spatial approximations were needed in case $1 \mathrm{GhGp}$, and the ODE matches simulations exactly in the limit of an infinite lattice. For case $2 \mathrm{LhGp}$, using $Q_{1 \mid 1}=2 / 3$ as obtained from pair approximations underestimates clustering of habitat and therefore underestimates population density; using the value $Q_{1 \mid 1}=0.8079$ measured from simulations in the ODEs yields very good agreement. For case $3 \mathrm{GhLp}$, the ODEs overestimate equilibrium population density, as is typically the case when using pair approximation with locally dispersing population models (Matsuda et al., 1992); the presence of spatially unstructured dynamic habitat does not change that behavior. Finally, for case 4 LhLp, with only local interactions, the underestimation of habitat clustering $Q_{1 \mid 1}=2 / 3$ leads to an underestimation of population density; using the more accurate value of habitat clustering then yields the typical overestimation of population density from pair approximation (due to underestimating the clustering of occupied sites).

Next, note that in population models on fixed landscapes, equilibrium density is a function of the fecundity and mortality parameters $\phi$ and $\mu$ only through their ratio $\phi / \mu$. To explore how this result differs in our model due to the additional presence of dynamic habitat, we again performed a parameter sweep where $\phi$ varied between 1 and 20; however, we also let $\mu=\phi / 10$ to keep a 


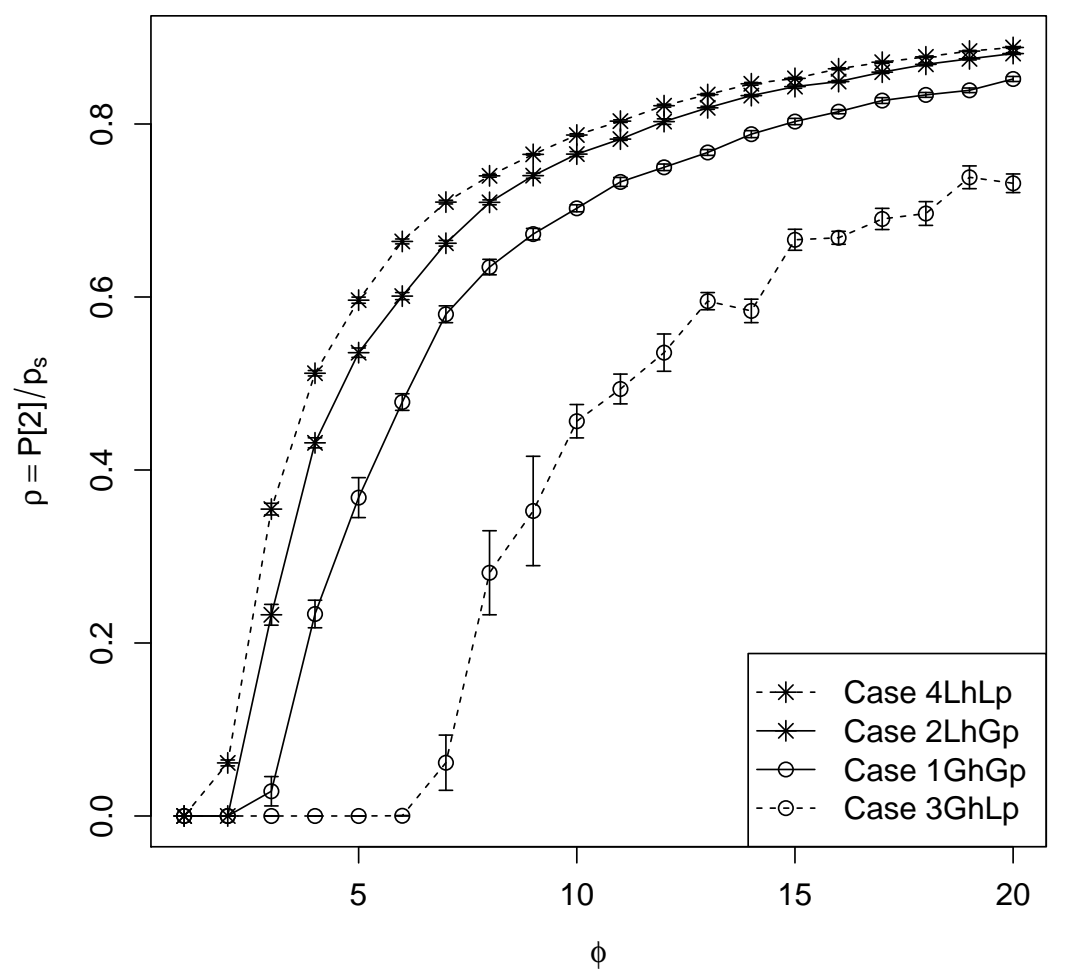

Figure 2: The equilibrium proportion of suitable sites occupied, $\rho^{*}=P^{*}[2] / p_{s}$, is shown as fecundity $\phi$ varies from 1 to 20 . Mortality was fixed at $\mu=1$. The figure legend is ordered to match the relative positions of the curves in the figure. 

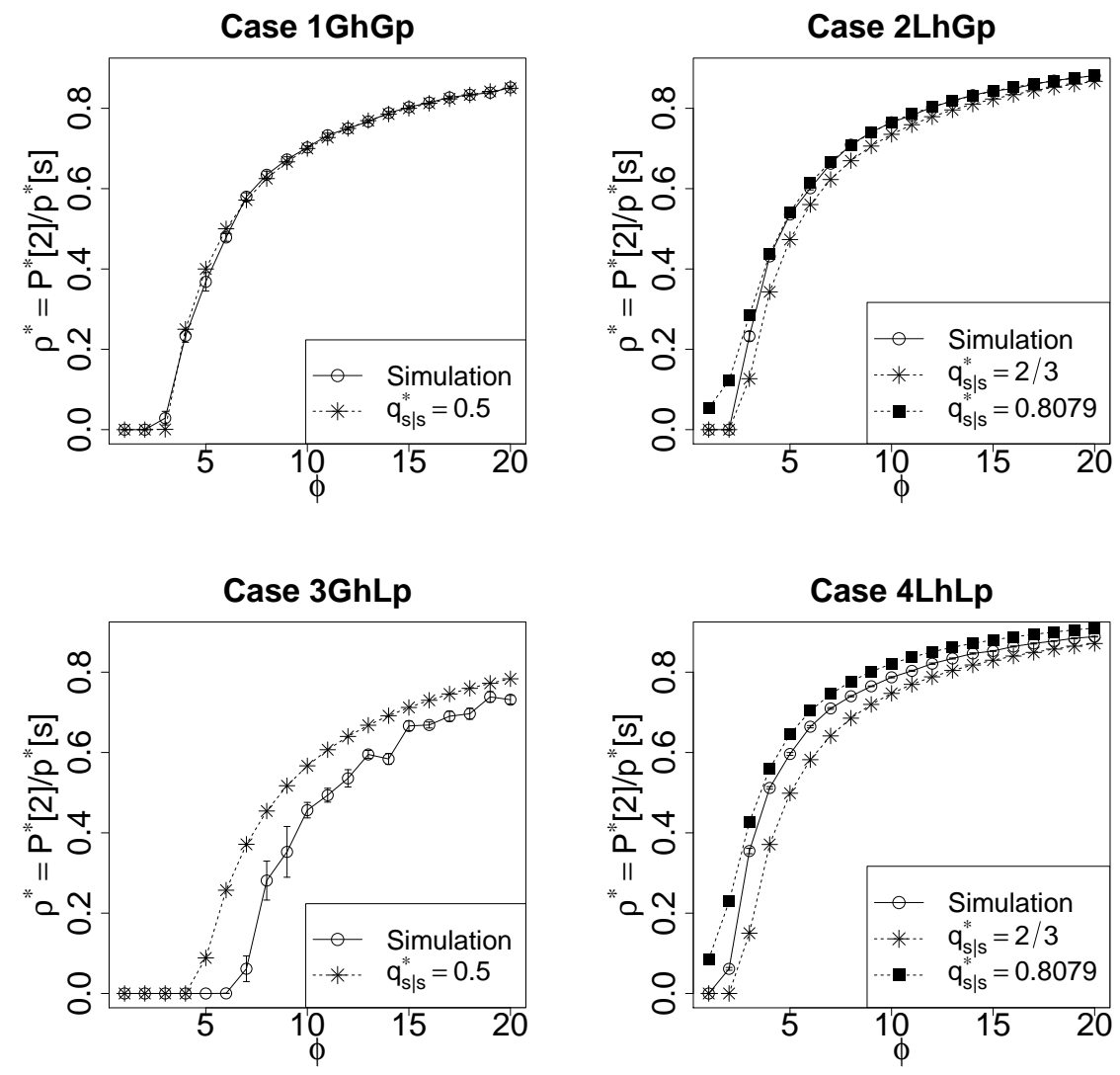

Figure 3: Results as in Figure 2, but with ODE results included as well. 


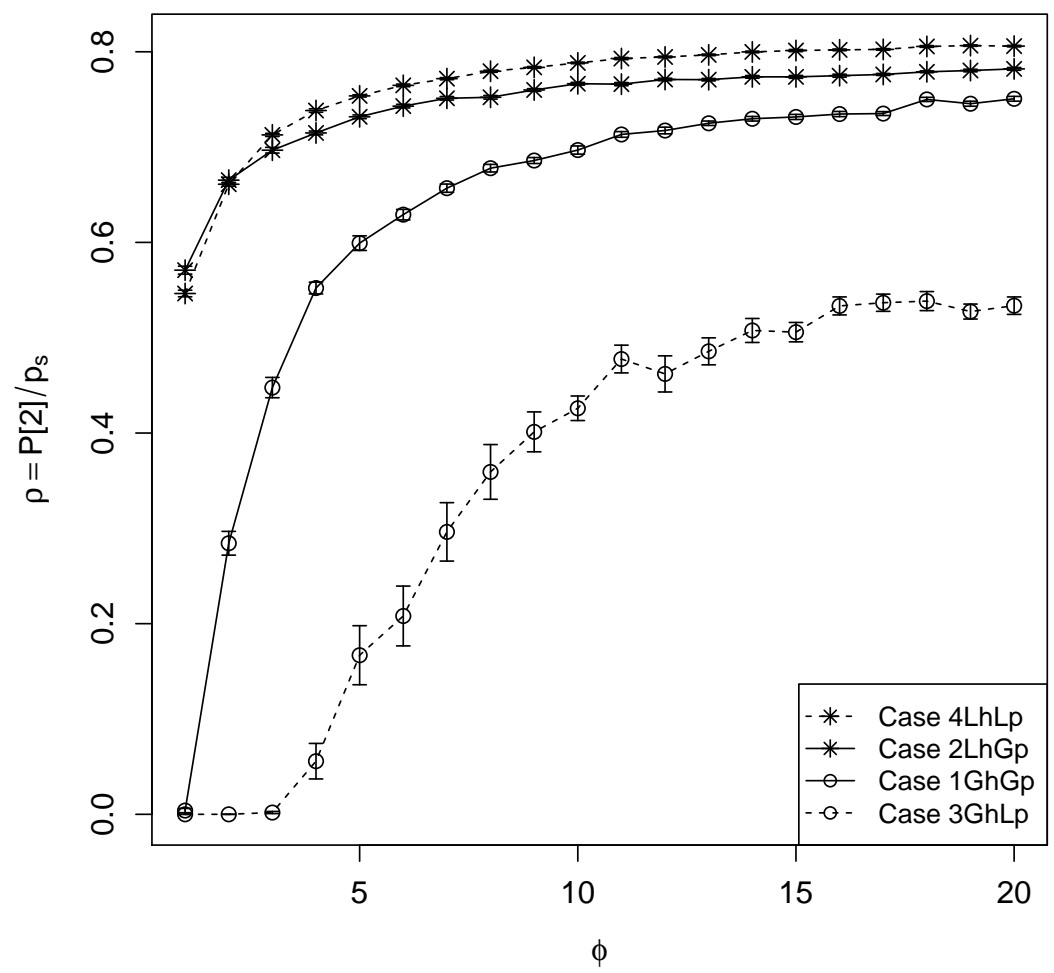

Figure 4: The proportion of suitable sites occupied, $\rho=P[2] / p_{s}$, is shown as fecundity $\phi$ varies from 1 to 20. Mortality was adjusted to keep a constant ratio $\phi / \mu=10$.

fixed ratio $\phi / \mu=10$. The results are shown in Figure 4. For large enough $\phi$, equilibrium density is essentially independent of the value of $\phi$, as population dynamics overwhelm habitat dynamics. However, as $\phi$ becomes smaller, eventually habitat dynamics begin to dominate and decrease population density.

Finally, we performed a double parameter sweep with $\phi$ varying between 0 and 20 and $\mu$ varying between 0 and 10, with 40 values each. We measured the equilibrium population density $\rho^{*}$ from among five replicate simulations for each parameter combination. For the $8000=40 \times 40 \times 5$ observations of 


\begin{tabular}{ccccc} 
Case & $\beta_{0}$ & $\beta_{1}$ & $\beta_{2}$ & $R^{2}$ \\
\hline 1GhGp & 0.9910437 & 1.0038078 & 0.5130016 & 0.993310 \\
2LhGp & 0.9856285 & 4.3238963 & 2.1829193 & 0.997674 \\
3GhLp & 1.0507575 & 0.9479394 & 0.2321650 & 0.976244 \\
4LhLp & 0.9787122 & 4.5287909 & 2.6741057 & 0.998141
\end{tabular}

Table 2: Results of fitting the model $\rho^{*}=\beta_{0}-\left(\beta_{1} \mu+0.5\right) /\left(\beta_{2} \phi\right)$ to simulation results as $\phi$ was varied between 0 and 20 , and $\mu$ was varied between 0 and 10 .

$\left(\rho^{*}, \phi, \mu\right)$ in each of the four cases, we fit a nonlinear least-squares regression model $\rho^{*}=\beta_{0}-\left(\beta_{1} \mu+0.5\right) /\left(\beta_{2} \phi\right)$ to obtain a descriptive relation between equilibrium population density and the fecundity and mortality parameters; the results are shown in Table 2. The model fit fairly well in all four cases, with the results for case $1 \mathrm{GhGp}$ matching their expected values from Equation (5) of $\beta_{1}=1$ and $\beta_{2}=0.5$.

\section{Discussion}

With spatial population models of this type, one of the primary challenges is to determine why a given set of results emerge; that is, what are the salient properties of the system that affect quantities of interest, such as population density or clustering? By examining four different cases with our model, we have explored several types of spatial structure and dynamics that can play important roles in populations on landscapes with changing habitat distributions.

With static habitat heterogeneity and a globally dispersing population, the spatial arrangement of suitable and unsuitable habitat has no effect on equilibrium population density due to the absence of spatial correlations in occupied sites (Hiebeler, 2004). In such a case, all suitable sites are equally likely to be occupied, regardless of the states of other sites in the neighborhood. If that 
were still true with our model, the population density would be identical in cases 1GhGp and 2LhGp (global population dispersal, with global and local habitat dynamics respectively). However, with dynamic habitat, the spatial scale of habitat dynamics does influence the density of even a population with global dispersal, because it affects the effective turnover rate of habitat. With localized habitat dynamics, sites tend to be surrounded by sites with the same habitat type, and therefore change habitat type more slowly as compared to the case with global habitat dynamics. This slowdown in effective habitat turnover leads to an increase in population density, even with global population dispersal. Also, for a globally dispersing population on a static heterogeneous landscape, all suitable sites on the landscape are equally likely to be occupied, with no spatial correlations in patch occupancy among adjacent suitable sites. In our case 2LhGp, however, even with global dispersal, there are still spatial correlations in patch occupancy among nearby suitable sites, due to the spatial dynamics (and resulting local spatial correlations) of habitat. Suitable sites near the edge of a patch are less likely to be occupied, because it is more likely that they were recently unsuitable.

Previous work by Matlack and Leu (2007) with dynamic landscapes found that segregating habitat turnover into fewer sites increased population density, compared with a case where habitat turnover was more widely distributed. In our model, when habitat dynamics are local, habitat turnover is also segregated into a smaller number of sites relative to the case of global habitat dynamics. Unlike the model of Matlack and Leu (2007), the set of particular sites capable of experiencing habitat turnover in our model with local habitat dynamics shifts over time as the distribution of habitat types evolves. That is, the segregation of disturbances is not done within fixed regions over time. But within any short interval of time, only sites near the boundary of suitable and unsuitable habitat experience turnover. 
Pair approximation in spatial population models tends to underestimate the amount of local clustering, and therefore overestimate population density, though various techniques can be used to build improved approximations, depending on the geometry of the lattice (Sato et al., 1994; Hiebeler, 1997; van Baalen, 2000). However, a pair approximation model of case 2LhGp (local habitat, global population) leads to a predicted equilibrium population density $\rho^{*}$ that underestimates the value as measured by simulations. We speculated that this was due to underestimation of the clustering of suitable habitat in the landscape. Replacing the pair approximated value $Q_{1 \mid 1}=2 / 3$ by the value $Q_{1 \mid 1}=0.8079$ measured from simulations confirmed this suspicion, as ODEs and simulations then agreed. When both types of interactions are local (case 4LhLp), pair approximation again underestimates population density. In that case, the clustering of suitable habitat and of occupied sites are both being underestimated, with the former error having a larger effect on the predicted population density than the latter. This is again seen when habitat clustering is based on simulation measurements rather than pair approximation.

In the model explored here, either local or global population dispersal may be more beneficial (in terms of increasing equilibrium population density $\rho^{*}$ ), depending on the spatial scale of habitat dynamics and of the resulting spatial correlations in habitat type. This type of response to habitat structure has also been observed in models with fixed habitat (Hiebeler, 2000). When habitat types are clustered, local population dispersal allows the population to exploit suitable patches (case 4LhLp versus case $2 \mathrm{LhGp}$ ); when habitat is not clustered, global dispersal allows the population to escape local crowding (case 1GhGp versus case $3 \mathrm{GhLp}$ ). In addition to changing the likelihood of dispersal into poor habitat and increasing edge effects, the fragmentation of suitable habitat may also lead to increased demographic stochasticity (North and Ovaskainen, 2007), although when the habitat fragmentation is dynamic, this effect is likely 
to be reduced relative to a system with static or slowly-changing habitat. The change in the spatial distribution of organisms with respect to other individuals and with respect to the quality of nearby habitat can have both positive and negative effects on population density (North and Ovaskainen, 2007).

The presence of habitat dynamics with its own intrinsic timescale also removes the invariance of the model with respect to changes in fecundity and mortality rates $\phi$ and $\mu$ with fixed ratio $\phi / \mu$. Now, even when $\phi / \mu$ is held constant, the relative rates of population dynamics versus habitat dynamics determines which type of dynamics will dominate the system. This phenomenon is also seen in a related model of populations on dynamic habitat where habitat dynamics are due to outside disturbances (Hiebeler et al., submitted).

In landscapes with static heterogeneous habitat, spatial clustering of habitat types can lead to the presence of isolated patches of suitable habitat surrounded by a matrix of unsuitable sites. Local dispersers cannot cross these barriers, leading to local extinction within patches. In systems with dynamic habitat, dynamic corridors can connect these otherwise separate patches, allowing the population to move from one patch to another (Wimberly, 2006; Kun et al., 2009), which can be more difficult on static landscapes, especially when suitable habitat is less frequent. The situation may be more complex with multi-species models, where dynamic corridors may affect species richness curves via increased persistence time, albeit with fewer species (Roy et al., 2004). The strength of fragmentation effects will also be strongly affected by the dispersal pattern of new offspring (Matlack and Leu, 2007).

By rescaling time, the results in Figure 4 could also be interpreted as showing equilibrium population density $\rho^{*}$ versus landscape turnover rate while keeping $\phi$ and $\mu$ fixed. This shows that $\rho^{*}$ typically decreases monotonically as the landscape/habitat turnover rate increases. Previous work by Banitz et al. (2008) showed that under some circumstances, biodiversity is maximized at in- 
termedidate disturbance rates, when the disturbances are spatially correlated. However, in their model, a disturbed site was immediately able to be recolonized; there were no lasting effects of disturbance as in our model when a site turns unsuitable. Kun et al. (2009) explored a locally dispersing population on a landscape with spatially uncorrelated dynamic habitat type; their model corresponds to our case $3 \mathrm{GhLp}$. In their study, they found that as long as $\mu>0$, equilibrium population density is maximized for an intermediate value of the landscape turnover/disturbance rate. However, the effect was very weak (with the change in $\rho^{*}$ becoming nearly monotonic) as the amount of suitable habitat $p_{s}$ increased. The curve corresponding to case 3GhLp in Figure 4 may indeed show that $\rho^{*}$ is maximized for an intermediate value of $\phi$. Differences in models details between this study (in continuous time) and that of Kun et al. (2009) (in discrete time) make it difficult to perform a direct comparison of results for given parameter values.

Many additional features could be added to the model explored here. For example, there could be two-way interactions between population and habitat, with populations affecting the dynamics of habitat fragments (del Castillo, 2015). For example, plant consumption by herbivores changes the distribution of plants (suitable habitat); it may even be possible for the quality of plants to evolve over time (de Mazancourt and Loreau, 2000). Dispersal distance could be made a function of local habitat suitability, as population dispersal has been shown to have interactive effects with habitat suitability and fragmentation (Radinger and Wolter, 2015). Directed dispersal toward more favorable habitat would allow the population to exploit spatial structure in habitat suitability even more strongly (Cantrell et al., 2006). Spatial scales in natural and human-driven systems of course does not follow a clear black-and-white distinction with local and global spatial scales, but instead involves interactions over a variety of intermediate scales. This model and related ones show that the 
relative spatial and temporal scales of population and environmental processes as well as disturbances (such as control measures) all come into play when trying to control the outbreak of invasive or undesirable species (Hiebeler, 2005; Hiebeler and Morin, 2007; Hiebeler et al., submitted). The spatial scale of measurements may affect observed population dynamics, including the amount of variability observed (Lundberg et al., 2000).

Underlying/intrinsic habitat dynamics such as those explored here may be due to natural processes such as forest gap formation (Kubo et al., 1996), where new gaps form far from other gaps ("global transitions") due to individual tree mortality or wind events, or adjacent to other gaps ("local transitions") due to the change in environmental conditions within an existing gap. They may also arise due to human influences, such as management of forest stands, where again transitions may be global, due to policy or advice at a regional scale, or local, due to the influence of nearby landowners (Huff et al., 2015). When using pesticides to control invasive species, the spatial and temporal scale of population dispersal and intrinsic habitat dynamics may interact to guide management strategies, including the spatial extent of disturbance events acting as control measured (Hiebeler et al., submitted).

\section{Acknowledgements}

This material is based upon work supported by the National Science Foundation under Grant No. DMS-0718786 to D.H.

\section{References}

Andrén, H., 1994. Effects of habitat fragmentation on birds and mammals in landscapes with different proportions of suitable habitat: A review. Oikos 71, $355-366$. 
Banitz, T., Huth, A., Grimm, V., Johst, K., 2008. Clumped versus scattered:

How does the spatial correlation of disturbance events affect biodiversity? Theoretical Ecology 1, 231-240.

Bodin, Ö., Tengö, M., Norman, A., Lundberg, J., Elmqvist, T., 2006. The value of small size: Loss of forest patches and ecological thresholds in southern Madagascar. Ecological Applications 16 (2), 440-451.

Bonin, M. C., Almany, G. R., Jones, G. P., 2011. Contrasting effects of habitat loss and fragmentation on coral-associated reef fishes. Ecology 92 (7), 15031512.

Cantrell, R. S., Cosner, C., Lou, Y., 2006. Movement toward better environments and the evolution of rapid diffusion. Mathematical Biosciences 204, $199-214$.

Chesson, P. L., 1985. Coexistence of competitors in spatially and temporally varying environments: A look at the combined effects of different sorts of variability. Theoretical Population Biology 28, 263-287.

Cleland, E. E., Collins, S. L., Dickson, T. L., Farrer, E. C., Gross, K. L., Gherardi, L. A., Hallett, L. M., Hobbs, R. J., Hsu, J. S., Turnbull, L., Suding, K. N., 2013. Sensitivity of grassland plant community composition to spatial vs. temporal variation in precipitation. Ecology 94 (8), 1687-1696.

Collinge, S. K., Palmer, T. M., 2002. The influences of patch shape and boundary contrast on insect response to fragmentation in California grasslands. Landscape Ecology 17, 647-656.

de Mazancourt, C., Loreau, M., 2000. Grazing optimization, nutrient cycling, and spatial heterogeneity of plant-herbivore interactions: Should a palatable plant evolve? Evolution 54 (1), 81-92. 
Debinski, D. M., Holt, R. D., Apr. 2000. A survey and overview of habitat fragmentation experiments. Conservation Biology 14 (2), 342-355.

del Castillo, R. F., 2015. A conceptual framework to describe the ecology of fragmented landscapes and implications for conservation and management. Ecological Applications 25 (6), 1447-1455.

DeWoody, Y. D., Feng, Z., Swihart, R. K., 2005. Merging spatial and temporal structure within a metapopulation model. The American Naturalist 166 (1), $42-55$.

Didham, R. K., Hammond, P. M., Lawton, J. H., Eggleton, P., Stork, N. E., 1998. Beetle species responses to tropical forest fragmentation. Ecological Monographs 68 (3), 295-320.

Diffendorfer, J. E., Gaines, M. S., Holt, R. D., 1995. Habitat fragmentation and movements of three small mammals (sigmodon, microtus, and peromyscus). Ecology 76 (3), 827-839.

Fahrig, L., 1992. Relative importance of spatial and temporal scales in a patchy environment. Theoretical Population Biology 41, 300-314.

Fahrig, L., Jul. 1997. Relative effects of habitat loss and fragmentation on population extinction. The Journal of Wildlife Management 61 (3), 603-610.

Hiebeler, D. E., 1997. Stochastic spatial models: From simulations to mean field and local structure approximations. Journal of Theoretical Biology 187, $307-319$.

Hiebeler, D. E., 2000. Populations on fragmented landscapes with spatially structured heterogeneities: Landscape generation and local dispersal. Ecology 81 (6), 1629-1641. 
Hiebeler, D. E., 2004. Competition between near and far dispersers in spatially structured habitats. Theoretical Population Biology 66 (3), 205-218.

Hiebeler, D. E., 2005. Spatially correlated disturbances in a locally dispersing population model. Journal of Theoretical Biology 232 (1), 143-149.

Hiebeler, D. E., 2007. Transient dynamics and quasistationary equilibria of continuous-time linear stochastic cellular automata voter models with multiscale neighborhoods. Advances in Complex Systems 10 (Supplementary issue 1), $145-165$.

Hiebeler, D. E., Houle, J., Drummond, F., Bilodeau, P., Merckens, J., submitted. Locally dispersing populations in heterogeneous dynamic landscapes with spatiotemporal correlations. I. Block disturbance. Journal of Theoretical Biology.

Hiebeler, D. E., Michaud, I. J., 2012. Quantifying spatial and temporal variability of spatially correlated disturbances. Ecological Modelling 240, 64-73.

Hiebeler, D. E., Morin, B. R., 2007. The effect of static and dynamic spatially structured disturbances on a locally dispersing population. Journal of Theoretical Biology 246 (1), 136-144.

Hodgson, J. A., Moilanen, A., Thomas, C. D., 2009. Metapopulation responses to patch connectivity and quality are masked by successional habitat dynamics. Ecology 90 (6), 1608-1619.

Hovel, K. A., Lipcius, R. N., 2001. Habitat fragmentation in a seagrass landscape: Patch size and complexity control blue crab survival. Ecology 82 (7), $1814-1829$.

Huff, E. S., Leahy, J. E., Hiebeler, D., Weiskittel, A. R., Noblet, C. L., 2015. An agent-based model of private woodland owner management behavior using 
social interactions, information flow, and peer-to-peer networks. PLoS ONE 10 (11), e0142453.

Jacquemyn, H., Butaye, J., Hermy, M., 2003. Influence of environmental and spatial variables on regional distribution of forest plant species in a fragmented and changing landscape. Ecography 26 (6), 768-776.

Johnson, D. M., 2004. Source-sink dynamics in a temporally heterogeneous environment. Ecology 85 (7), 2037-2045.

Keymer, J. E., Marquet, P. A., Vlasco-Hernández, J. X., Levin, S. A., Nov. 2000.

Extinction thresholds and metapopulation persistence in dynamic landscapes. The American Naturalist 156 (5), 478-494.

Kimura, M., Weiss, G. H., Apr. 1964. The stepping stone model of population structure and the decrease of genetic correlation with distance. Genetics 49 (4), 561-576.

Kubo, T., Iwasa, Y., Furumoto, N., 1996. Forest spatial dynamics with gap expansion: Total gap area and gap size distribution. Journal of Theoretical Biology 180, 229-246.

Kun, Á., Oborny, B., Dieckmann, U., 2009. Intermediate landscape disturbance maximizes metapopulation density. Landscape Ecology 24, 1341-1350.

Lande, R., Oct. 1987. Extinction thresholds in demographic models of territorial populations. The American Naturalist 130 (4), 624-635.

Limberger, R., Wickham, S. A., 2012. Disturbance and diversity at two spatial scales. Oecologia 168, 785-795.

Loehle, C., Mar. 2007. Effect of ephemeral stepping stones on metapopulations on fragmented landscapes. Ecological Complexity 4 (1-2), 42-47. 
Lundberg, P., Ranta, E., Ripa, J., Kaitala, V., 2000. Population variability in space and time. Trends in Ecology and Evolution 15 (11), 460-464.

Matlack, G. R., 2005. Slow plants in a fast forest: Local dispersal as a predictor of species frequencies in a dynamic landscape. Journal of Ecology 93, 50-59.

Matlack, G. R., Leu, N. A., Dec. 2007. Persistence of dispersal-limited species in structured dynamic landscapes. Ecosystems 10 (8), 1287-1298.

Matlack, G. R., Monde, J., 2004. Consequences of low mobility in spatially and temporally heterogeneous ecosystems. Journal of Ecology 92, 1025-1035.

Matsuda, H., Ogita, N., Sasaki, A., Sato, K., 1992. Statistical mechanics of population. Progress of Theoretical Physics 88 (6), 1035-1049.

Molofsky, J., Bever, J. D., 2002. A novel theory to explain species diversity in landscapes: Positive frequency dependence and habitat suitability. Proc. R. Soc. Lond. B 269, 2389-2393.

Molofsky, J., Durrett, R., Dushoff, J., Griffeath, D., Levin, S., 1999. Local frequency dependence and global coexistence. Theoretical Population Biology $55,270-282$.

Moloney, K. A., Levin, S. A., 1996. The effects of disturbance architecture on landscape-level population dynamics. Ecology 77 (2), 375-394.

Munguia, P., Osman, R. W., Hamilton, J., Whitlatch, R., Zajac, R., 2011. Changes in habitat heterogeneity alter marine sessile benthic communities. Ecological Applications 21 (3), 925-935.

North, A., Ovaskainen, O., Jul. 2007. Interactions between dispersal, competition, and landscape heterogeneity. Oikos 116 (7), 1106-1119. 
Radinger, J., Wolter, C., 2015. Disentangling the effects of habitat suitability, dispersal, and fragmentation on the distribution of river fishes. Ecological Applications 25 (4), 914-927.

Renshaw, E., 1991. Modelling Biological Populations in Space and Time. Cambridge University Press.

Roy, M., Pascual, M., Levin, S. A., 2004. Competitive coexistence in a dynamic landscape. Theoretical Population Biology 66, 341-353.

Santos, R. O., Lirman, D., Pittman, S. J., 2015. Long-term spatial dynamics in vegetated seascapes: Fragmentation and habitat loss in a human-impacted subtropical lagoon. Marine Ecology.

Sato, K., Matsuda, H., Sasaki, A., 1994. Pathogen invasion and host extinction in lattice structured populations. Journal of Mathematical Biology 32, 251268.

Schelling, T. C., 2006. Micromotives and Macrobehavior. W.W. Norton \& Company, Inc.

Sherratt, T. N., 2006. Spatial mosaic formation through frequency-dependent selection in Müllerian mimicry complexes. Journal of Theoretical Biology $240(2), 165-174$.

Snäll, T., Ehrlén, J., Rydin, H., 2005. Colonization-extinction dynamics of an epiphyte metapopulation in a dynamic landscape. Ecology 86 (1), 106-115.

Uriarte, M., Bruna, E. M., Rubim, P., Anciães, M., Jonckheere, I., May 2010. Effects of forest fragmentation on the seedling recruitment of a tropical herb: Assessing seed vs. safe-site limitation. Ecology 91 (5), 1317-1328. 
van Baalen, M., 2000. Pair approximations for different spatial geometries. In: Dieckmann, U., Law, R., Metz, J. A. (Eds.), The Geometry of Ecological Interactions. Cambridge University Press, Ch. 19, pp. 359-387.

Weiss, G. H., Kimura, M., 1965. A mathematical analysis of the stepping stone model of genetic correlation. Journal of Applied Probability 2, 129-149.

Wiegand, T., Revilla, E., Moloney, K. A., Feb. 2005. Effects of habitat loss and fragmentation on population dynamics. Conservation Biology 19 (1), 108-121.

Wimberly, M. C., 2006. Species dynamics in disturbed landscapes: When does a shifting habitat mosaic enhance connectivity? Landscape Ecology 21, 35-46.

\section{Appendix A. Derivation of ODEs}

The general ODE for $P[00]$ in all of the cases is as follows:

$$
\begin{aligned}
\frac{d P[00]}{d t}= & P[01] r_{[01] \rightarrow[00]}+P[10] r_{[10] \rightarrow[00]}+P[02] r_{[02] \rightarrow[00]}+P[02] r_{[20] \rightarrow[00]} \\
& -P[00]\left(r_{[00] \rightarrow[01]}+r_{[00] \rightarrow[10]}+r_{[00] \rightarrow[02]}+r_{[00] \rightarrow[20]}\right)
\end{aligned}
$$

where $r_{[i j] \rightarrow[k \ell]}$ gives the rate at which a pair of sites in states $[i j]$ transitions to the state pair $[k \ell]$. For all cases, $r_{[02] \rightarrow[00]}=r_{[20] \rightarrow[00]}=\mu$ due to intrinsic mortality.

For cases $1 \mathrm{GhGp}$ and $3 \mathrm{GhLp}$ with global habitat dynamics,

$$
r_{[01] \rightarrow[00]}=r_{[10] \rightarrow[00]}=p_{s}=P[0]+P[2]
$$

as unsuitable becomes suitable at a rate equal to the proportion of suitable habitat in the entire landscape. For cases $2 \mathrm{LhGp}$ and $4 \mathrm{LhLp}$ with local habitat dynamics,

$$
r_{[01] \rightarrow[00]}=r_{[10] \rightarrow[00]}=\frac{z-1}{z} Q_{s \mid 10}+\frac{1}{z}
$$


where the first term give the rate of the type- 1 site in the pair turning suitable due to one of the $z-1$ neighbors outside of the pair being suitable (with $Q_{s \mid 10}=$ $\left.Q_{0 \mid 10}+Q_{2 \mid 10}\right)$ and the second term gives the rate of the type-1 site turning suitable due to its known suitable neighbor within the pair.

Similarly, for cases $1 \mathrm{GhGp}$ and $3 \mathrm{GhLp}$,

$$
r_{[00] \rightarrow[01]}=r_{[00] \rightarrow[10]}=P[1],
$$

while for cases $2 \mathrm{LhGp}$ and $4 \mathrm{LhLp}$,

$$
r_{[00] \rightarrow[01]}=r_{[00] \rightarrow[10]}=\frac{z-1}{z} Q_{1 \mid 00}
$$

where in the latter expression, there is no $1 / z$ term because there is no unsuitable site in the pair to turn the other site unsuitable.

Finally, for cases $1 \mathrm{GhGp}$ and 2LhGp with global population dispersal,

$$
r_{[00] \rightarrow[02]}=r_{00] \rightarrow[20]}=P[2] \phi .
$$

${ }_{534}$ For cases $3 \mathrm{GhLp}$ and $4 \mathrm{LhLp}$ with local population dispersal,

$$
r_{[00] \rightarrow[02]}=r_{00] \rightarrow[20]}=\frac{z-1}{z} Q_{2 \mid 00} \phi
$$

35 due to colonization by one of the $z-1$ neighbors outside of the pair.

536 The general ODE for $P[01]$ is

$$
\begin{aligned}
\frac{d P[01]}{d t}= & P[00] r_{[00] \rightarrow[01]}+P[02] r_{[02] \rightarrow[01]}+P[11] r_{[11] \rightarrow[01]}+P[21] r_{[21] \rightarrow[01]} \\
& -P[01]\left(r_{[01] \rightarrow[00]}+r_{[01] \rightarrow[11]}+r_{[01] \rightarrow[21]}\right)
\end{aligned}
$$

As8 with the ODE for $P[00]$, for all cases, $r_{[21] \rightarrow[01]}=\mu$ due to intrinsic mortality.

39 The rates $r_{[00] \rightarrow[01]}$ and $r_{[01] \rightarrow[00]}$ are the same as in Equations (A.1)-(A.4) above. 


$$
r_{[02] \rightarrow[01]}=P[1] .
$$

${ }_{542}$ For cases 2LhGp and 4LhLp,

$$
r_{[02] \rightarrow[01]}=\frac{z-1}{z} Q_{1 \mid 20} .
$$

${ }_{543}$ For cases $1 \mathrm{GhGp}$ and $3 \mathrm{GhLp}$,

$$
r_{[11] \rightarrow[01]}=p_{s}=P[0]+P[2] .
$$

${ }_{544}$ For cases 2LhGp and 4LhLp,

$$
r_{[11] \rightarrow[01]}=\frac{z-1}{z} Q_{s \mid 11}=\frac{z-1}{z}\left(Q_{0 \mid 11}+Q_{2 \mid 11}\right) .
$$

${ }_{545}$ For cases $1 \mathrm{GhGp}$ and $3 \mathrm{GhLp}$,

$$
r_{[01] \rightarrow[11]}=P[1] .
$$

${ }_{546}$ For cases 2LhGp and 4LhLp,

$$
r_{[01] \rightarrow[11]}=\frac{z-1}{z} Q_{1 \mid 01}+\frac{1}{z}
$$

${ }_{547}$ where the last term is the rate at which the known unsuitable site in the pair

${ }_{548}$ causes the known suitable site to become unsuitable.

${ }_{549}$ Finally, for cases 1GhGp and 2LhGp,

$$
r_{[01] \rightarrow[21]}=P[2] \phi .
$$

550 For cases $3 \mathrm{GhLp}$ and $4 \mathrm{LhLp}$,

$$
r_{[01] \rightarrow[21]}=\frac{z-1}{z} Q_{2 \mid 01} \phi .
$$

